\title{
Sergio E. Serrano: Engineering uncertainty and risk analysis: balanced approach to probability, statistics, stochastic modeling, and stochastic differential equations
}

\author{
Published 2011 by HydroScience Inc., Ambler, USA; ISBN 978-0-9655643-1-1, \\ 462 pp (available from Amazon.com, paperback US \$193)
}

\section{Ronny Berndtsson}

Published online: 26 May 2011

(C) Springer-Verlag 2011

Uncertainty and risk analyses are becoming more and more important in engineering sciences due to increasing climatic variation and other types of natural hazards. On average, every year infrastructure damages caused by earthquakes, floods, typhoons and other catastrophic events induce costs corresponding to hundreds of billions of dollars besides thousands of lives lost. Consequently, uncertainty and risk analyses are some of the most important subjects for engineers. In line with this, the author, Prof. Sergio E. Serrano, Temple University, has issued a second, completely revised text book on the subject.

The book is a comprehensive collection of stochastic and statistical engineering techniques with uncertainty and risk analyses that are applicable in a broad range of problems. The focus is clearly on engineering applications rather than on theoretical derivation. Even rather advanced techniques involving nonlinear differential equations are solved analytically in supplied codes and presented with illustrative examples. The strong and very useful aspect of this text book is that it contains a multitude of solved examples as well as problems with answers. Consequently, the potential reader of this book might be engineering students as well as professional engineers.

The book consists of fourteen chapters starting with a discussion of engineering uncertainty analysis and ending with a summary and discussion on seven steps for analysis of engineering problems. Chapter 2 introduces concepts of probability and frequency. Chapter 3 clarifies the concept of random variables with discrete and continuous variables. Chapter 4 then introduces the basics of random system modeling and Monte Carlo simulation. Chapter 5 presents the concept of several random variables and Chapter 6 introduces basic ideas of statistics. Chapter 7 shows how to fit probabilistic models to experimental data and then follows linear regression in Chapter 8. Chapter 9 explains how to develop models for reliability of engineering systems and Chapter 10 shows how to design engineering experiments. In Chapter 11 methods for testing experimental data are introduced together with ANOVA techniques. Chapter 12 is a synthesis of previous chapter in the way that it explains and visualizes random processes using explained Maple codes. Finally, Chapter 13 introduces novel techniques to solve linear and nonlinear differential equations by means of analytical methods.

The above shows that it is a very comprehensive text book on uncertainty and risk. This together with solved examples and included Maple codes makes it one of the most useful books on the market for teaching engineering students as well solving practical engineering problems involving risk and uncertainty estimation. I am sure that the book will have a significant positive impact on improving engineering solving during coming decades. 